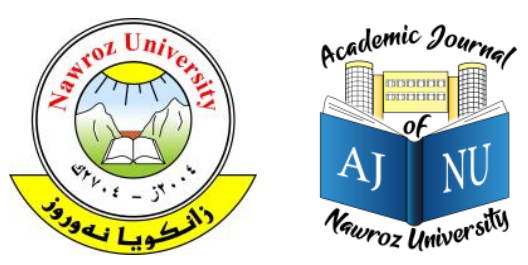

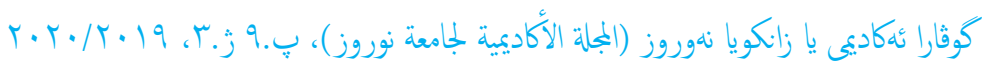

حقوق الطبع والنشر (2017. هذه مقالة الوصول اليها مفتوح موزعة تحت رخصة

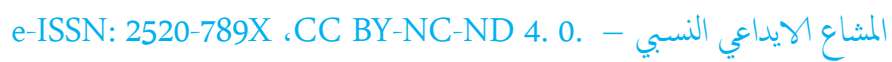

https://doi.org/10.25007/ajnu.v9n3a793

\title{
حل التناقض بين الاستغلال والاستكشاف
}

\section{(معالجة نظرية)}

دئاري محمد علي، كلية التقنية الادارية، جامعة دهوك التقنية، اقليم كردستان العراق

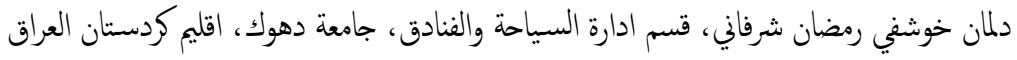

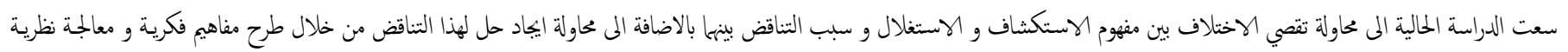

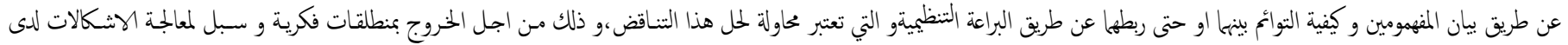

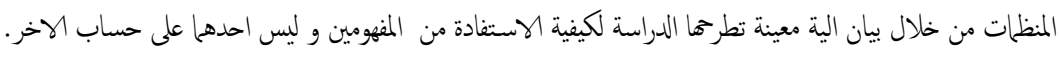

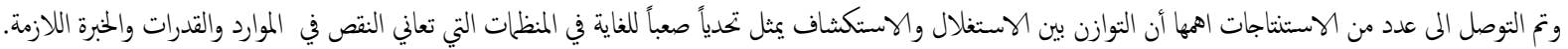

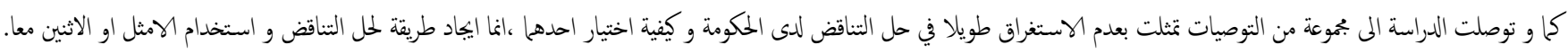

الكلمات المثتاحية: حل التناقض، الاستغلال، الاستكشاف.

1

تحديداً الأعال المختلفة تتراوح بين النغير التكنولوجي إلى تصميم المنظمة (شرفاني،

حيث ان معظم الباحثين السابقين الذين كتبوا عن الموضوع يتعاملون مع الاستغلال والاستكشاف على أنَّم عنصرين من عناصر الموابجة ( Confronting (Elements)، (O’Reilly \& Tushman, 2013: 3وف يحاول الباحثان في هذه الدراسة السعي المى ايياد حل للمعضلة الانفة الذكر و توجيه المنظلات الحكومية اللى قرار حتمي يتعلق بسير عمليانها دون التخبط و التوقق و ضياع الوقت على الخلاف حول البعدين و تحديد اولوية احدها على الاخر.

\section{2 - 2 المبحث الأول: منهجية الدراسة}

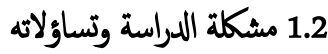

ان الظروف الحالية التي يمر بها الاقليم من ازمة مالية وقلة التخصيصات والنقد من قبل الحكومة المركزية و بسبب التوجيهات الحالية من قبل رئاسة الأقليم بالحد من النفقات والصعوبات الموجودة في كيفية الايفاء بالالتزامات المالية واككمال المشاريع وتقديم الخدمات المى المجتع ودفع الرواتب والمستحقات أدت الى ظهور مشاكل كيرة
الاستكشاف و الاستغلال مفهومان متناقضان و هناك صعوبة في معرفة المدراء لكيفية الموائمة بين الاثنين تفضيل احدها على الآخر.قدمت العديد من النظريات الإدارية المعاصرة ظواهر تنظيمية ضمن فئات منفصلة ومتناقضة منّمَا دفح المنظلات للتركيز على إمّاّ الاستغلال أو الاستكشاف في حين يشير (March) أنَّ المنظلات بحاجة إلى الإنياز إلى كل من الاستغلال والاستكشاف لان التزكيز أُحادي الجانب على الاستغلال يُمكن أن يعزز الأداء قصير الأجل ولكن يُمكن أن يؤدي إلى حُ، الكفاءة، لأنَّ المنظمات قد لاتكون قادرة على الاستجابة بصورة كافية لتغيرات البيئة، وعلى عكس ذلك الكثير من الاستكشاف يُمكن أن تعزز قدرة المنظمة على تجديد قاعدها المعرفية لكن تضع المنظات في دوامة لانهاية لها من الدراسة والتغير غير المجزي، إنَّ بقاء المنظمة على المدى الطويل ونجاحا تعتمد على قدرتها على تلى المشاركة في الاستغلال مايكفي لضمان بقاء المنظمة حالياً والمشاركة في الاستكشاف بما يكفي لضمان البقاء في المستقبل، كما أن المنظات الناجحة هي التي ساهت البراعة في تحولها العام في البحوث التنظيمية من المفاضلة إلى التفكير المتناقض، وبشكل أكثر 


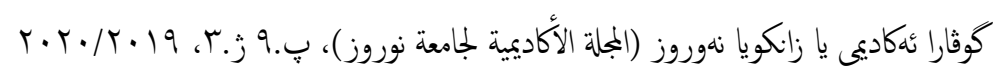

(Karrer, Karrer, 2013:7). حيث ان هنالك العديد من المسوغات العلمية التي ترتبط بأهمية التفكير بشكل متناقض والتعامل بغعالية مع التناقض. إلا ان التساؤل المهم والذي من المفترض الإجابة عليه في هذا الدراسة يتثثل في الأتي: - - ماهي طرق حل التناقض بين الاستغلال والاستكشاف؟

\section{2}

تنطلق أهمية الدراسة من ضرورة توافر حلول لمعالجة التناقض بين الاستغلال والاستكشاف، ويكن تحديد أهميته من خلال الأتي:

السعي العلمي لبناء نموذج يين فيه طرق حل التناقض بين الاستغلال والاستكشاف بما يؤدي الى الكفاءة في العمل الاداري للحكومة.

قلة الدراسات الاجنبية وعدم وجود اية دراسة عربية تطرقت الى محولة حل التناقض بين الاستغلال والاستكشاف.

محاولة الوصول الى آلية للتوازن بين الاستكشاف و الاستغلال .

\section{2}

إن الهدف من هذا الدراسة يشمل اجراءات الاستكتشاف النظري لبعض الدراسات الحديثة، التي اجريت مؤخرا على حل التناقض بين الاستغلال والاستششاف، لتفسر عن العلاقة بنهها بطريقة مطوية من خلال توفير المبادئ التوجيية لإجراء المزيد من الابحاث، ومعالجة الفجوات التي تركها البحوث السابقة، وذلك من خلال تقديم فكرة عن كيف يتمن المديرين ان يتعاملوا بفعالية في العمل،

من خلال سلسلة من التقنيات والاليات المرتبطة بأدبيات التناقض. بناء الإطار النظري الخاص بهذا الدراسة كمحاولة لتقديم أهم ما توصلت إليه الأدبيات والدراسات في موضوع معالجة التناقض بين الاستغلال والاستكشاف.

التعرف على طرق حل التناقض بين الاستغلال والاستكشاف.

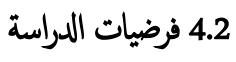

تنبثق فرضية الدراسة من خلال خلفة الدراسة النظري، حيث تنص على أن "هناك مجموعة من الطرق التي تقدم حلولاً نطرياً لمعالجة التناقض بين للاستغلال والاستكاف،و بالتالي يمكن للمنظمات من ان تخصص مواردها بين الانشطة
جدا لدى الحكومة بالدرجة الاولى و بالتالي انعكس اثرها على اداء المنظات الحكومية .

بالاضافة الى ذلك و في دراسة قامت بها (الشرفاني، 2015)، اشارت النتائج في الدراسة الانفة الذكر إلى أن إجابات المبحوثين وعلى المستوى الكلي للعينة المبحوثة لبعد الاستغلال تميل باتجاه الاتفاق على توافره لديهم وبنسبة (85.15\%)كما و اشارت النتأُ التي توصلت الهيا الشرفاني إلى أنَّ إجابات المبحوثين وعلى المستوى الكلي للعينة المبحوثة حول بعد الاستكشاف تميل باتجاه الاتفاق على توافره لدهم بنسبة (88.6\%) من تلك الإجابات . أي ما زلنا لانستطيع اتباع اي المتغيرين، وبما ان الدراسة العلمية هو اكلمال مسيرة الباحثين الاخرين، ارتأينا ان نذهب ابعد من توضيح المفهومن الى ايياد طريقة لحل التناقض واستخدام المزيج الامثل. إن كيفية حل التناقض بين أنشطة الاستغلال والاستشكاف للمنظات يعد موضوعاً

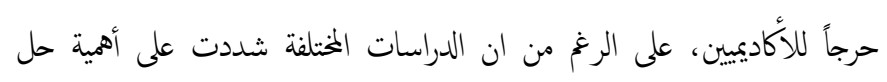
التناقض بين الاستغلال والاستكشاف في نفس الوقت، فقد كرست القليل من المناقشات لمسألة كيف يككن للمنظات التوافق بين المطالب المتضاربة للاستغلال والاستكشاف (Jansen, 2005: 36). وعلى الرغ من أن هناك إتفاق بين الأراء على ضرورة تحقيق التوازن بين الاستغلال والاستكشاف، إلا أن هناك القليل من التوضيح عن كيفية إنشاء هذا التوازن

(Gupta, et al., 2006: 697)

و جاءت هذه الدراسة كمحاولة لمساعدة المدراء و الختصين في الحكومة في كيفية توجيه موارد الدولة المالية والمخصصات (النثرية) و الاستفادة منها بشكل كفوء و ونه فعال من خلال توضيح الية معينة للمسوؤلين في كيفية صرفها سواءاً على الاستغلال او الاستكشاف.

ان فكرة التناقض يمكن ان تقوم بهمة التوفيق فيا يتعلق بالتعامل مع الصراعات والثناقضات المعاكسة، الكامنة في استكثاف واستغلال، توجد هذا التوافق في طبيعة تركية متعددة المستويات، وتكمن العلاقة في جوهر التزكيبة على المستوى التنظيمي، وتتطلب المنظات ان تكون بارعة في معالجة التوترات بنهها في وقت واحد، بالاضافة المى ذلك، التفكير المتناقض يناسب مع الحلات التي عندما لايوجد هناك خيار فعال يككن حل التوتر لان هناك حاجة الى حلول معارضة ومتشابكة 


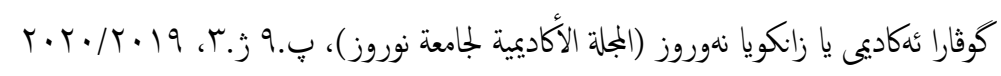

تم طرح فكرة حل التناقض بين الاستغلال والاستكشاف بشكل متناسق ومستمر عبر عدة مداخل في نظرية المنظمة، الإدارة الإستراتيجية، والإقتصاد

Holmqvist, 2004: )، (Winter \& Szulanski, 2001: 732) الإداري

إن مفهوم التناقض يوفر اطارا شاملاً التي يكن ان يفسر المفاهيم المتعلقة بالاستكشاف والاستغلال كالتوتر والمفاضلة او المعضلة، والمفاهيم التي تشكل في Karrer, Karrer, ) المستويات الدنيا مثل وحدة الاعمال والادارات الفنية

تم وصف العلاقة بين الاستغلال والاستكاف بوصفها عمليات مترابطة التي تحتاج إلى الجمع والتضمين لتوليد النتاجُ المساعدة، أنَّ المنظات تميل إلى تقسيم إهتماتها ومواردها بين الاستكشاف والاستغلال (شرفاني، 2015: 32). ويكن توضيح طرق الحل بينها كالآتي:

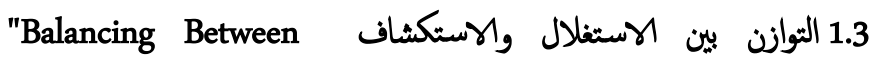
Exploration and Exploitation" إن تحقيق التوازن بين الانشطة الاستغلالية والاستشافية كانت أحد الاسئة الرئيسية في أبحاث الإدارة (Chang \& Hughes, 2012: 13). حيث إن التوازن بين الاستغلال والاستكشاف يشكل تحدياً حاساً وصعباً للغاية في المنظات التي تنتقر إلى الموارد والقدرات والمبرة اللازمة لتنفيذ البراعة بنجاح، وتعتبر المشكلة الأساسية التي تواجه المنظلات لكي تصبح بارعة هي إيجاد التوزان المستقيم بين الاستغلال والاستكشاف (Yigit, 2013:8). ينشأ التوازن ما بين الاستغلال والاستكشاف من الحساسية تجاه البيئة والمحافظة على هيكل تنظيمي متوافق، والذي بدوره يؤدي إلى طول العمر التنظيمي (Organizational longevity)، المنظلات التي تكون قادرة على بناء قدرات جديدة وفي نفس الوقت استخدام القدرات الموجودة تتمتع بمستويات أعلى من الأداء الإستراتيجي للمشروع من خلال إنشاء الإبداع عن طريق الأستثار في التقنيات، المنظلات الموجه للتكنولوجيا ترتبط بجولات متتالية أو دورية من الاستغلال والاستكشاف ومجزة بشكل أفضل لمتابعة إبداعات المنتج، وتحسين أداء وحدات O’Reilly \& Tushman, ) الأعال لإبداعات المنتج من خلال عملية الإبداع .(2004: 79
الاستغلالية و الاستكثافية واحدها على حساب الاخر. وان حل هذا التناقض بين الاستكشاف والاستغلال سوف يؤدي الى سهولة اتخاذ القرارات المتعلقة بتخصيص الموارد للمنظمة بشكل كفؤه وفعال.

5.2 الدراسة الحالية هي دراسة نظرية أعتمدت على التحليل المهنجي المعمق للأدبيات العلمية المرتبطة بالاستكشاف والاستغلال ومن ثم مناقشتها والعمل على استخلاص المفاهيم الأساسية التي نعمل على وضعها في أطار علمي منهجي من خلال صياغة أنموذج يحتوي على طرق حل التناقض بين الاستغلال والاستكشاف مبنية فيها جميع الطرق التي تؤدي الى معالجة حل التناقض بينها.

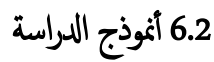
تم اعتاد أسلوب التحليل النظري المهنجي لحل التناقض بين الاستكشاف والاستغلال التي تتضمن حلول ثانوية لكل منها وتأثير كل منها على نتابُ عمل المنظمة . 3. المبحث الثاني: حل الثناقض بين الاستغلال والاستكشاف قبل عرض حل التناقض بين الاستغلال والاستكشاف لابد من عرض مفهوم الاستغلال والاستكشاف وكما يلي: يككن تعريف الاستكشاف بأنه التزكيز الاستراتيجي على الاحتياجات المستقبلة، يعني الاختلاف بدلا من الموثوقية، والبحث عن الجديد وتوسيع المسح البيئي بدلا من بحث وثيق والمسح البيئي الحلالي، وانتاج معرفة جديدة بدلا من توسيع المعرفة الحالية، الابداع الراديكلي(الجذري) او المتقطع بدلا من التغير المستمر، والعقود الاجلة البديلة الممكنة من شأهها أن تؤدي الى نتائج معروفة. أما الاستغلال التركيز الاستراتيجي على احتياجات الزبائن الحالية، يعني الموثوقية على الاختلاف، والبحث والتوثيق والمسح البيئي الحالي بدلا من البحث وتوسيع المسح البئي، وتوسيع ماهو معروف حاليا وليس مايكنن ان يكون معروفا، التغيير المستمر على الابداع الجذري (Bodwell, 2011: 64). بينت الدراسات السابقة أن الاستغلال والاستكشاف يحتاجان إلى معارية مختلفة He \& wong, 2004: ) وكفاءات مختلفة التي يمكن أن تنشأ تحديات متناقضة Benner \& ) ،Gibson \& Birkinshaw, 2004: 216) ،487 .(Tushman, 2003: 245 


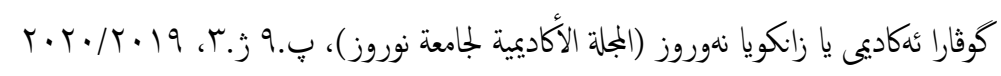

Gibson \& ) ،(OReilly \& Tushman, 2011: 18) المعكسين في المنظطات

.(birkinshaw, 2004: 220

$$
1 \text { الجدول } 1
$$

الاقتزاحات الرئيسة للاستغلال والاستكشاف لإدارة النناقض 1

\begin{tabular}{|c|c|c|c|c|}
\hline الباحث الرئيسي & الامتزاح الرئسي & كيفية ادارة & الافتراض & المهدخل \\
\hline $\begin{array}{c}\text { (Jansen, et } \\
\text { al.,2009) } \\
\text { (Kauppila, 2010) } \\
\text { (Tushman \& } \\
\text { O’Reilly,1996) } \\
\text { (Birkinshaw \& }\end{array}$ & تركز إثقافة وحدات العمليات التمال مخلفة & الفصل المهاني & الازدواجيات المنشة، & الهيكلية \\
\hline $\begin{array}{c}\text { Gibson, 2004) } \\
\text { (Carmeli \& } \\
\text { Halevi, 2009) } \\
\text { (Gibson \& } \\
\text { Birkinshaw, } \\
\text { 2004) }\end{array}$ & 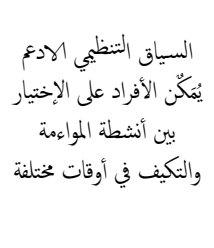 & الفصل المؤقت & الازدواجيات & السياقية \\
\hline $\begin{array}{c}\text { (Boumgarden, et } \\
\text { al., 2012) } \\
\text { (Siggelkow \& } \\
\text { Levinthal, 2003) }\end{array}$ & 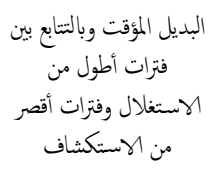 & الفصل المؤقت & الأزمدواجتقات، & المؤرة \\
\hline $\begin{array}{c}\text { (Andriopoulos \& } \\
\text { Lewis, 2009) } \\
\text { (Fredberg, 2014) } \\
\text { (Heracleous \& } \\
\text { Wirtz, 2014) } \\
\text { (Martini, et al., } \\
\text { 2013) } \\
\text { (Smith, et al., } \\
\text { 2010) } \\
\text { (Westenholz, } \\
\text { 1993) }\end{array}$ & 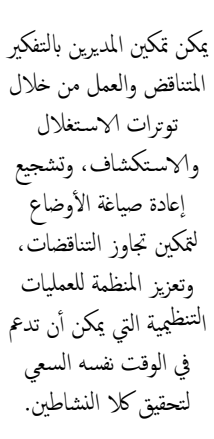 & المابعد الفصل الزمال النماني & 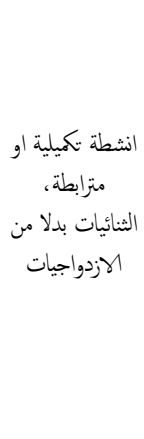 & التناقض \\
\hline
\end{tabular}

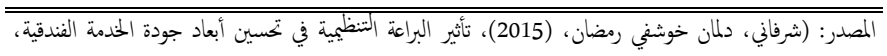

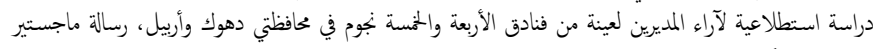

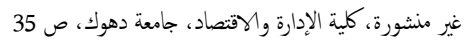

إن الجمع بين الاستغلال والاستكشاف يرتبط مع بقاء المنظمة لفتزة أطول، وفي مدرد الدراسات التجريبة وجدت أن المنظلات التي توازن بين الاثنين تكون أقل عرضة

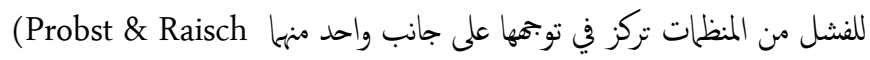
.(He \& Wong, (Birkinshaw \& Gibson, 2004: 220) ،, 2005: 93)

2004: 483) وان احد الصراعات الهامة لحل التناقض بين الاستغلال والاستكشاف يأتي عندما يتم الجمع ما بين الأمد الطويل والأمد القصير والدمج بين المعرفة الفردية والجماعية وفي هذا المعني فإن الاستراتيجيات والإجراءات المفيدة للمنظات على المدى الطويل ليست دائماً مفيدة على المدى القصير ، وبالمثل الأنشطة والعناصر التي تكون جيدة
بالإضافة إلى ذلك اشار (O’Reilly \& Tushman, 2011: 19) إلى سرعة التكيف مع البيئة المتغيرة وكذلك محدودية الموارد في المنظات وكيف أن الحاجة المى المنتجات قد يجبر المنظات للنعامل مع الانشطة الاستغلالية والاستكشافية في وقت واحد، من قبل الوحدات الفرعية المستقلة والإستراتيجيات والناذج وتعديلات مختلفة داخل نفس المنظمة لكل الأبعاد المتضاربة. وبالتالي يتطلب الحاجة إلى التوازن بين الاستغلال والاستكشاف، علاوة على ذلك تعتبر البراعة بأهها القدرة على إدارة هذه الأبعاد المختلفة للتكيف التنظيمي لبيئة متغيرة (Yigit, 2013:12). في دراسة (March, 1991) بين أن التحدي الرئيسي للمنظمة هو حول الموازنة بين

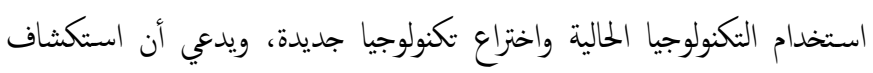
تكنولوجيا، اسواق، بدائل جديدة يضعف سرعة وأداء المهارات الحالية التي تم

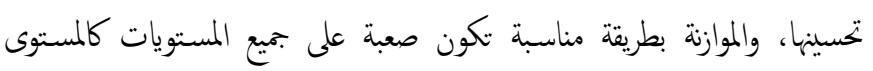
الفردي، المستوى التنظيمي، ومستوى النظام الإجتاعي، وان إيجاد التوزان بين Yigit, ) الاستغلال والاستكشاف تقع تحت موازنة عمليات التباين والاختيار .(2013: 25 هناك عدد قليل من المنظات التي تستطيع ان تحقق توازن بين الاستكشاف والاستغلال، خاصة مع زيادة النعقيد والضغوط لكي تظل قادرة على المنافسة، عادةً المنظمات تكون معرضة للتناوب لدع الأتجاه التكنيكي على حساب الإبداع الاستراتيجي, وبشكل خاص ينطبق هذا مع المنظمات التي تكلخ من أجل تقديم أعالها الإعتيادية عندما تحصل عليها في وقت قصير، الأموال، المنتج، والجودة غالباً ما ينظر إلى استكشاف مصادر جديدة بإعتبارها مشروع محفوف بالخاطر إلى المجهول وبدلاً من الخطوة التي تؤدي إلى تعزيز العمل (Bot, 2012: 23). يلخص الجدول (1-1) المدخل الأساسي في الأدبيات والاقتراحات والافتراضات الرئيسية بالنسبة لهذين النوعين من الانشطة .إن نظرية التناقض تؤكد انه من خلال تقديم ان النشاطين وبمرور الوقت يككن ان تكون مكملة ومترابطة بشكل ديناميكي، وعلى افتراض انهم في حالة من الازدواجية، والمحافظة على خيار النكلمل وكما مبين في الجدول 1. هذه الانشطة المتداخلة تحتاج إلى أن يتم ادارتها في المنظمة في وقت واحد، وتعتبر البراعة واحدة من القدرات الديناميكية للمنظلات بسبب أستخدام كلا المدخلين 


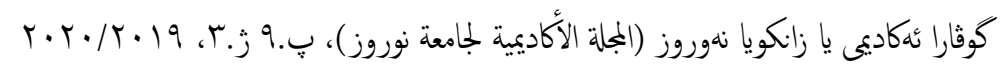

يؤدي إلى الصراعات والتناقضات والتضارب، والمارسات التنظيمية والإجراءات هي أسبقيات قابالة للأستمرار لتوازن متناسق (Simsek, 2009: 603).

\section{"Cyclical" 2.1.3 الثوازن بشكل دوري}

نوع من التوازن في المنظات التي ترتبط في فترات طويلة من الاستغلال (أو الاستقرار النسبي) عن طريق حلقات متفرقة من الاستكشاف (أو التغيير) والأمر يتعلق بالتوازن النقطي، وتوجد سوابق للبراعة بشكل دوري في ممارسات الموارد

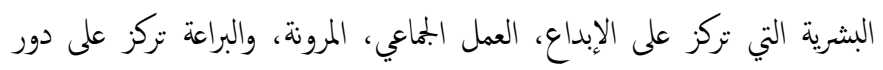
الهيكل المزدوج من خلال مبادرات التنفيذ نظرية المنظمة (5atta, 2011: (D).

3.1.3 التوازن بشكل جزئي "Partitional يشار إلها بإعتبارها ظاهرة مترابطة ومتزامنة في وقت واحد، والتي تنطوي على تجزئة ومزامنة الاستغلال والاستكثاف داخل الوحدات الهيكلية الخختلفة للمنظمة، من وبجة نظر هيكلية تتحقق البراعة بشكل جزئي من خلال إنشاء وحدات أو أقسام منفصلة للاستغلال أو الاستكشاف، مع كل وحدة تجسيد أنظمة المنطق الإستراتيجي والتشغيلي المتميز والثقافات والقدرة على متابعة ننائج كل من الاستغلال والاستكشاف في نفس الوقت من استضافة هياكل متعددة ومتناقة والعمليات والثقافات داخل نس المنظمة، في حين تتكامل على مستوى وحدة الأعال، يجب أن تبقى هذه المناطق المتباعدة عبر وحدات الأعال، بالإضافة إلى إستخدام الشبكات الخارجية، في دراسة أجريت على ما يقارب (20.000) تحالفات خلال فترة عشر سنوات، لوحظ أن يككن متابعة كلا الاستغلال والاستكثاف عبر ثلاث مجالات من التحالفات الاستراتيجية بما في ذلك وظيفة سلسلة القيمة من التحالفات وسات الخاصة بالشركاء في التحالف وموقف الشركاء في التحالف

.(Simsek, et al., 2009: 871)

\subsection{3 التوازن بواسطة المبادلة "Reciprocal":}

يحدث عندما تصبح مخرجات الاستغلال من وحدات الاعمال مدخلات لوحدة أعمال ثانية ومخرجات وحدة الأعمال الثانية مرة أخرى تصبح مدخلات لوحدة الأعال الثانية وهكذا بشكل دورة متكملة، إن التبادل المستمر للمعلومات بين الوحدات

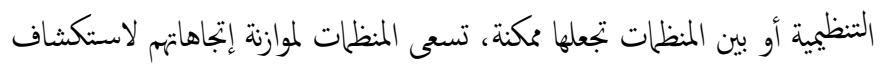
واستغلال ما يتعلق بطبيعة تخالفاتهم أو إختيار الشركاء مع مرور الوقت وعبر المجالات، وتسليط الضوء على النتائج التي توصلت إليها (5atta, 2011:).
في المدى القصير قد لانكون جيدة في المدى الطويل، ماهو جيد لجزء من المنظمة قد لاتكون جيدة لجزء من آخر أو نظام آخر (Yigit, 2013: 26). وإستناداً إلى الباحثين وتجارهم الخاصة، ينبني إيجاد التوزان الصحيح الذي يكن أن تخدم بشكل أفضل أغراض منظمته (Surendra, 2012: 2). إن أننطة الاستغلال والاستكشاف ضرورية للمنظات، لكنها تتنافس على الموارد النادرة، ونتيجة لذلك يجب على المنظلات إتخاذ خيارات صريحة وضنية بين الأثنين، في هذه الحالة المنظلات تتناج إلى أن تكون على علم بهياكلها التنظيمة فإنها تحتاج إلى أن تكون قادرة على أستشعار التغيرات والفرص المتاحة في السوق، وبالتالي تحديد الإجراءات التي يحتاجونها لإتخاذ وإعادة تكوين النظام الخاص بهم

للتكيف المستدام (Yigit, 2013: 8).

قدم (Raisch \& Birkinshaw, 2008 :389) حلولاً لحل متطلبات التناقض وبين أن الخروج بأنشطة استغلالية أو استكشافية يكون إما عن طريق الأستعانة بعادر خارجية أو عن طريق إقامة تحالفات، وهذه الحلول لما قواسم مشتركة بأن المنظات تقوم بحل التناقض من خلال المشاركة فقط في نشاط واحد في كل مرة وعلى العكس من ذلك البراعة التنظمية تتطلب من المنظلات معالجة الاستغلال والاستكشاف في وقت واحد، وقد ركزت الدراسات على ثلاثة مناجج واسعة داخل

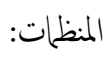

الحلول الهيكلية التي تسمح لنشاطين يتم تنفيذها في الوحدات التنظيمة المختلفة.

$$
\text { الحلول السياقية التي تسمح النشاطين أن تتابع في نس الوحدة. }
$$

الحلول المستندة على القيادة التي تجعل فريق الإدارة العليا هو المسؤول عن

$$
\text { التوفيق بين الأستجابة للتوترات بين النشاطين. }
$$

يمكن تحديد العديد من الطرق التي تحقق التوازن بين الاستغلال والاستكثاف تيف Simsek, et al., ) وفي ضوء مراجعة الدراسات والأبحاث الإدارية ذات العلاقي :(Datta, 2011: 5) ،(Simsek, 2009: 603)، (2009: 871

"Harmonic" 1.1.3 الثوازن عن طريق الثوافق ) ويتم تحقيق توازن توافقي من خلال تنفيذ الاستغلال والاستكشاف في الوقت نضسه في وحدة تنظمية واحدة، مثل هذا التوزان يتنافس على الموارد النادرة مما 


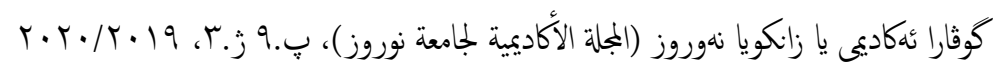

وفقاً لنظرية التعلم التنظيمي الفرق بين الاستكشاف والاستغلال أعتبر دائماً تناقضاً في الأدبيات (March, 1991)، من ناحية أخرى هناك العديد من الباحثين يدعون أن التفاعل بين الاستغلال والاستكثاف أنها لاتقتصر دائماً بندرة الموارد، هناك أنواع أخرى من الموارد مثل المعلومات والمعرفة والمهارات التقنية التي يمكن

استخداما للاستكشاف والاستغلال في نفس الوقت (Yigit, 2013:14). إن أساليب قرار التناقض تلعب دوراً رئسياً في وظيفة التوافق وينظر إليه كأحد المواضيع القادمة (Eisenhardt, 2000). Smith \& Lewis 2011: ( الأدبيات التنظيمة وهذا الموضوع أشارت إليه دراسة

وقد ناقش (Jansen, 2005) أن المنظلات التي تواجه هذه المعضلة قد تقوم بـ:

1.2.3 قبول التناقض من خلال الاستعانة بصادر خارجية Accept the paradox by compromising or outsourcing" المنظات قد تقبل التناقض لمواصلة الاستكشاف والاستغلال في نس الوقت، والتوصل إلى حل وسط بين هذين النشاطين، فيا يتعلق بالتطور النظري وقبول التناقض يذكر الباحثين أنه من أوجه عدم الاسساق وتمكنم من القيام بدراسة جدلية بين معارضة المستويات التي يتم التقاطها في النظريات المختلفة، وجد الباحثين أن أعضاء نجاح سلسلة الرباعيات (string quartets) كانت تدرك جيداً التوترات والتعرف على المفارقات (مثلاً بين الرغبة في الاستقلال الشخصي والقيادة القوية) مع ذلك كانت واعية لتجنب مناقشاتها، بدلاً من ذلك قد تفهم المنظلات التوترات بين الاستغلال والاستكشاف إما/أو وتطور الهيكل التنظيمة الميكنيكية أو العضوية، ومع ذلك نتتقد ان المعارضة بين كلا النشاطين لايككن حلها داخل المنظمة. بدلاً من ذلك هذه المنظلات قد تستعين بمصادر خارجية لجانب واحد من التناقض، وعملية شراء نتائج معينة إما من الاستغلال أو الاستكشاف من أطراف خارجية

.(Jansen, 2005: 37)

"Resolve the 2.2.3 اعادة حل (معالجة) التناقض من خلال الفصل المكاني paradox by spatial separation"

الفصل المكاني يشير إلى تزامن انشطة الاستكثاف والاستغلال في المنظمة، ومن الأمثلة النموذجية تقديم وحدة أعحال أساسية المسؤولة عن توليد والتقاط القيمة من المنتجات والأسواق القائم، في حين أن وحدة أعحال أخرى جديدة أو موجودة
إن أهمية التحالفات والشبكات الخارجية تتمثل بإعتبارها آليات للجمع بين الاستغلال والاستكشاف عبر الوقت والوحدات، وفي هذا السياق فإن تكمل المعرفة بين شركاء التحالف قد تكون ذات أهمية خاصة لقدرة المنظات التي تستخدم البراعة في أعالها للتوصل إلى منتجات جديدة جذرية دون إن يعيق ذلك الأسواق القائة وجعلها طوية الأمد، المنظلات التي تبقى لفترة طوية تمتلك عدة أوجه للتشابهات الهيكلية والثقافية، والعوامل التي تؤثر على البراعة تشمل اللامركزية وأسلوب إدارة Simsek, et al., 2009: (الضغوط والحساسية تجاه الاتجاهات الناشئة في البيئة

\section{3}

هذا المحور يستكشف في ضوء أدبيات التناقض التنظيمي، ويناقش بعض الأثار وييب أن تتساوى التوترات الكمنة وراء الاستغلال والاستكشاف مع فكرة التناقض (Smith \& Lewis, 2011:392). التناقض هي نتيضين تحدث في وقت واحد، ويكن وصفها بأهها الميل لحل التناقض من خلال أختيار واحد عكس الآخر او محاولة تحقيق التوازن بدرجات مختلفة وهذا يعني أن تطبيق النقيضين معاً في وقت واحد، مما يتيح للتوتر القيام بذلك وصولاً إلى Bunge, (Bodwell, 2011:47) الإبداع والبصيرة وزيادة الطاقة (بيبن

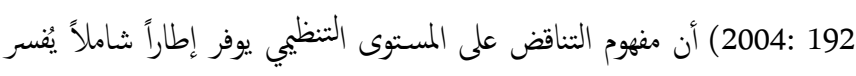
المفاهيم المتعلقة بالاستكشاف والاستغلال كالتوتر، المفاضلة، لغز، والمعضلة، بالإضافة إلى المفاهيم التي تتشكل في المستويات الدنيا مثل وحدة الأعمال والإدارات الفنية (Karrer \& Fleck, 2013: بالإضافة إلى ذلك الثفكير المتناقض يتناسب مع حالات عدم وجود خيار فعال يكنه حل التوتر لأن هناك حاجة إلى حلول متعارضة ومتشابكة ( LUscher \& Lewis, .(2008: 235 ان حل التناقض يتطلب النعامل مع التناقضات المعاكسة مثل الدراسة عن الجديد وتحسين القديم، الإبداع مقابل التكرار، النباين مقابل الإحتفاظ بها، المخاطرة مقابل الكفاءة وغيرها (Smith \& Lewis, 2011: 392). كذلك الصراعات الكمامنة المرتبطة مع الإدراك الإداري غير المتناسق، والسياقات التنظيمية غير المتناسقة، والمهارات الإدارية غير المتناسقة، معدلات التعلم، والتنافس على الموارد ( Karrer .(\& Fleck, 2013: 7 


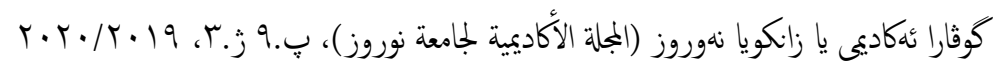

"Resolve the paradox by مل التناقض من خلال الفصل الزماني

temporal separation"

الفصل الزماني (المؤقت) يعني أن المنظمات قد تتعامل مع الاستغلال والاستكشاف عن طريق الفصل المؤت في الوقت المناسب ( De Bruijn, 40 :2011). المنظلات البارعة تضع الهياكل التنظمية المزدوجة التي تتناوب بين الاستغلال والاستكشاف على مر الزمن، ووجد أن وحدة القرار نفها تستخدم هياكل ميكانيكية لأتخاذ القرارات الروتينية، ثخ تحول إلى الهياكل العضوية لإتخاذ القرارات الغير روتينية، مثل هذه التقلبات المؤقتة يؤدي إلى تناسب أفضل بين هيكل المنظمة وهام ومتطلبات البيئة (Jansen, 2005:39.ويككن للمنظات أن تحل التناقض من خلال فصل الاستغلال والاستكشاف بشكل مؤقت (على أساس الزمن) تحقيق الاستغلال خلال فترة زمنية معينة وبالتالي تحقيق

الاستكشاف خلال فترة زمنية مختلفة (Benner \& Tushman, 2003: 247) حيث يتم إنجاز نفس المجموعة من موارد الاستغلال والاستشاف في فتزات زمنية مختلفة، وهذا يناشى مع ما اقترحه (Simsek, et al., 2009:870 من خلال العمل عن طريق التدخل في تركيز دوري للمنظمة على الاستكشاف والاستغلال، وقد عززت البراعة من خلال الأهنام المتتابع (المتسلسل) للأهداف.

Solve the paradox by " 4.2.3 حل التناقض من خلال التوازن

"balancing

اعترف الباحثين مؤخراً بقدرة وحدات الأعال لتحقيق التوازن في وقت واحد بين التوترات المتناقضة (Gibson \& Birkinshaw, 2004)، ( Jansen, 2005) 39). المنظلات البارعة وبهذه الطريقة قد تقوم بإنشاء الوحدات التنظيمة التي تتابع الاستغلال والاستكثاف في وقت واحد وتجمع بين ميزات الهياكل العضوية Sheremata, 2000: ) والميكانيكية، وبين الطاقات الدافعة والطاقات الجاذبة

هذه الوحدات التنظيمية تجمع بين مختلف العناصر المتناقضة مثل العمليات المادية وغير المادية والنظم والمهام الروتينة وغير الروتينية والطاقات الجاذبة والطاقات النابذة، هذه العناصر المتناقضة ينبغي ان تشجع المديرين لإجراء الإنشطة الاستغلالية والاستشافية في نفس الوقت ومع مرور الوقت تحول المنظات تركيزها من الاستقرار أو مواصلة الإبداعات الإضافية إلى الإبداع الجذري، عنا تتبع
ستكون مسؤولة عن استكشاف أسواق جديدة وتطوير التكنولوجيا الجديدة وتتبع الأتجاهات الناشئة في الصناعة (Birkinshaw \& Gibson, 2004: 218). وفي Portfolio ( بعض الأحيان يتحقق الأنفصال عن طريق استخدام مدخل المخفظة Approach الاستغلال والاستكشاف، تنفيذ الفصل المكني يتطلب مقايبس تتعلق بتخصيص الموارد لتلائم طبيعة أنشطة الاستكشاف والاستغلال، بينا المقايس التي تركز على الهدف تناسب الجهود الاستغلالية والمقايس التي يحركها الاكتشاف تناسب Karrer \& Fleck, ) الجهود الاستكشافية، التي تميل إلى التزكيز على التعلم .(2013: 8 وعلى النقيض من قبول التناقضات المنظلات يمكن أن تقوم بحل التوتر بين الاستكشاف والاستغلال عن طريق توضيح مختلف المستويات المرجعية والاتصالات فيا بينه من خلال متابعة الاستغلال والاستكشاف في نفس الوقت وفي ختلافة الأجزاء (الأقسام، الوحدات) في المنظمة، قد يحدث الفصل المكاني حسب المستوى أو الوظيفية أو الموقع، ويتميز بالإختلافات في الاستغلال والاستكشاف التي تتعلق بالمناصب الهرمية، وترتبط مختلف الأدوار الإستراتيجية للمديرين في العمليات الفرعية للتجديد الإستراتيجي والعمليات الفرعية الثلاثة أي Floyd \& ( تعريف الكفاءة، وتعديل الكفاءة ونشر الكفاءة تتميز بأدوار إدارية محددة Benner ) ،(Tushman \& O’Reilly, 2013: 9) ،(Lane, 2000: 159

.(\& Tushman, 2003: 242

الوحدات التنظمية التي تواصل الاستكشاف يتوقع أن تكون صغيرة ولامركزية مع ثقافات وعمليات طليقة والوحدات التنظيمة التي تتابع الاستغلال يتوق أن تكون أكبر وأكثر مركزية، مع ثقافات وعمليات ضيقة، الفصل الوظيفي عادةً يُستخدم في المنظات الكبيرة مع عمليات متعددة، (Benner \& Tushman, 2003: 247). وعلى سبيل المثال قسم الإنتاج عادةً ما يستغل الكفاءات والمهارات الحالية وتهدف إلى الكفاءة وانخفاض التكليف والوظائف الأخرى مثل التسويق أو الدراسة والتطوير هي موبحة نحو الاستكشاف والتجربة بهدف تطوير المنتج، وإيجاد زبائن وأسواق جديدة ) (Jansen, 2005: 38-39). 


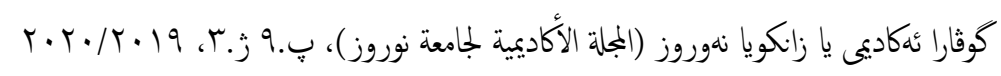

الجدول 2

التعامل مع تناقض الاستكشاف والاستغلال في المنظات

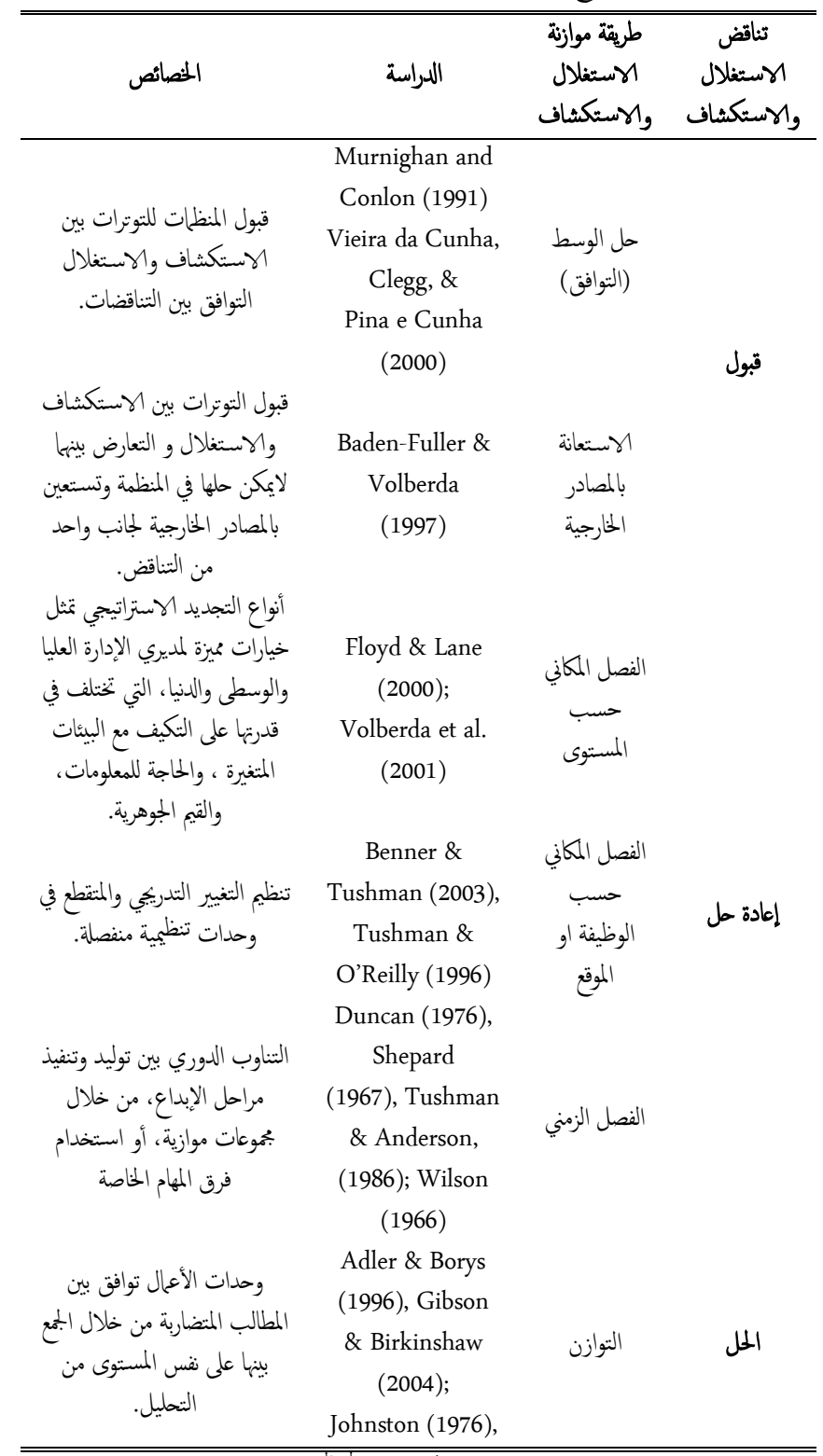

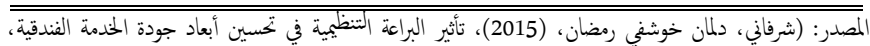

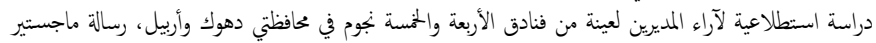

غير منشورة، كلية الإدارة والاقتصاد، جامعة دهوك، ص الارن 38).

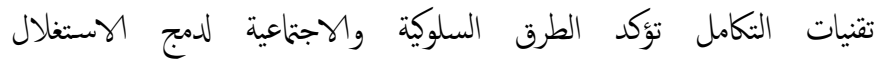
والاستكشاف، إن الفكرة الأساسية هنا هي أن التنشئة الإجتاعية، والموارد البشرية وفرق البناء، وتعزيز القيم المشتركة وتنسيق النعاون، مما يساعد الجهات الفاعلة على التفكير والتصرف بشكل بارع وعلى أساس يوي، وهذا المدخل تح وصفه عادةً على المستوى التنفيذي المرتبطة بالإدارة العليا، ويككن ترجمتها بأنها النكامل السلوكي، حيث يقوم فريق الإدارة العليا بمزامنة المرؤوسين العمليات الإجتاعية

والمهمة (Karrer \& Fleck, 2013:
المنظلات الإبداعات الإضافية أو المستقرة يكون هناك المزيد من التزكيز على الاستغلال، وعندما تتبع الابداعات الجذرية فيكون تركزها على الاستكشاف تهرت (Gibson \& Birkinshaw, 2004: 219) أو تطوير سياق تنظيم جاعي وفقاً لذلك حيث تتكون من العناصر التنظيمية المتناقضة، وادارة التوتر من خلال البيئات التنظيمية المعقدة. إن وحدات العمل تستخدم هياكل عديدة في وقت واحد، للتعامل مع مجموعة متنوعة من الحالات الطارئة التي توابجها، والباحثين وجدوا بمحوعة مختلفة من الأبعاد الهيكلية التي تشير إلى أن فكرة وجود هيكل واحد ثابت غير دقيق نسبياً في المنظلات البارعة لكيفية تحقيق التوازن بين الاستغلال والاستكشاف على مستوى الوحدات التنظيمة من شأنها أن تعزز فهمنا لماذا تختلف المنظلات البارعة في قدرتها على خلق قيمة من الاستكشاف والاستغلال، على الرغز من أن الدراسات السابقة قد اقترحت أن حل التناقض تؤدي إلى مستويات أعلى من الأداء، والتي قد توثر على النتائج اللاحقة من حيث الأداء الملالي، وبعبارة أخرى هذه الطرق Jansen, المتعددة قد تكون وسيطة في العلاقة بين البراعة التنظيمة الأداء المالمي ليني (2005: 39 ان التعامل مع العلاقة بين الاستكشاف والاستغلال يتطلب الفصل بين الاقطاب المتعارضة، وهو ما يطلق عليه بالبراعة المعارية (Gupta, et al., 2006: 701) Andriopoulos \& Lewis, ) ((Birkinshaw \& Gibson, 2004: 218) 698 (Karrer \& Fleck, 2013: 8)(2009: . ويثير هذا الأسلوب الى استخدام الهياكل والاستراتيجيات المزدوجة، المى جانب الجهود التي تزكز على Smith \& ) (Puranam et al., 2006: 270)(استكشاف والاستغلال 393 :Lewis 2011). ويكن توضيح ماذكر أعلاه من خلال الجدول 2.

\section{"Ambidexterity By Means Of التوازن من خلال تقنيات النكلمل 5.2.3}

Integration Tactics" عادةً ما ترتبط تقنيات النكمل مع الجوانب السلوكية لفرق الإدارة، التي قد تدمج كلا القطبين لعلاقة الاستغلال والاستكشاف، من خلال الإستفادة من إجراءات محددة أو القيم أو الأحكام (Andriopoulos \& Lewis 2009: 698)، (Gibson \& birkinshaw, 2004: 218) ،(Gupta, et al., 2006: 701) 


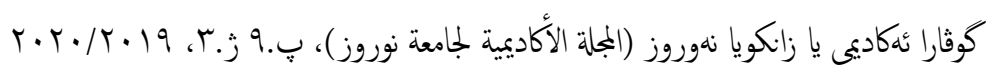

تواجه المنظلات صعوبات في المحافظة على التوازن بين الاستكشاف

والاستغلال.

إن المنظلات التي تعاني النقص في الموارد والقدرات والخبرات اللازمة يمثل التوازن بين الاستغلال والاستكشاف فيها تحدياً صعباً للغاية. ضرورة ميل المنظات الى تحديد اولوياتها في استخدام المصادر، وترجيح الكفة

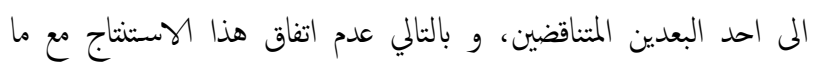
طرحه (march)

إن عملية حل التنافض هي القدرة الاساسية الكامنة وراء فعالية تنفيذ هذه المداخل وتحديد عندما يتفوق احد المداخل على الاخر في سلسلة متصلة من

الهعال استنادا الى (Tushman \& Orally).

ان فكرة معالجة التناقض كالمفاضلة والموائمة تشير المى انها حقيقة ناشئة لم يتم الجزم بها لحد الان في الدراسات التي نشرت والتي استطاع الباحثان

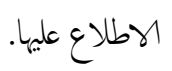
يتيح الفصل المكاني اجراء انشطة الاستغلال والاستكشاف في أماكن مختلفة

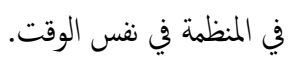

يمكن أن يآتي حل التناقض بين الاستغلال والاستكشاف عندما يتم الجمع ما بين المدى الطويل والمدى القصير وفي هذا المعني فإن الاستراتيجيات والإجراءات المفيدة للمنظات على المدى الطويل ليست دائماً مفيدة على المدى لئ القصير ،ولهذا يظهر التناقض بين انشطة كل من الاستغلال والاستكشاف. هناك أنواع أخرى من الموارد مثل المعلومات والمعرفة والمهارات التقنية التي تؤدي الى لى التناقض يككن استخداهما للاستكشاف والاستغلال في نفس الوقت.

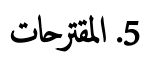

ضرورة قيام المنظمة بحل التناقص بين البعدين المتعارضين و محاولة استخدام و تطبيق احدها بما يتفق مع مصاد ر المنظمةو ليس استراتيجيانها، لان الاهداف الخططة قد لايمكن تحقتقها بالمصادر المتواجدة و كفية استخلالها ، و هذا ماحصل و يحصل مع مشكة الاقليم في الوقت الحالي.
ومساعدة المديرين التنفيذين على التعامل مع العمليات المعرفة المتناقضة للاستغلال Lubatkin, et ) ،Andriopoulos \& Lewis, 2009: 698) والاستكشاف (al., 2006: 651 ومن ثم تدع العمليات الإجتاعية (أي الإعتراف بالمارسات، والتنشئة الإجتاعية) والثقافة والعلاقات الشخصية، قد تساعد القوى الفاعلة التفكير والتصرف بشكل بارع (Smith \& Tushman, 2005: 527)، (Gibson \& Birkinshaw, 2004: 219). تتضمن الطرق الأخرى التي يكن أن يتحقق هذا التكمل إدخال ثقافات متناقضة أو الرؤية التنظيمية. 6.2.3 حل التناقض من خلال التتابع والتزامن أثار (Bot, 2012: 23) الى ملخص شامل للتطبيقات المتزامنة والمتتابعة لتعميم الاستغلال والاستكشاف، وثم يقترن كل مدخل مع بيئة الاعمال الاكثر ملائمة لها.وعلى الرغز من ان كل مدخل قد يبدو عكس ذلك في الطبيعة، و في الواقع تمثل نهايات متواصلة حيث يمكن ان تتواجد التطبيقات داخل هذه الحدود. في مدخل التنابع ينظر الى انشطة الاستكشاف والاستغلال كالانشطة المتعارضة جذرياً، وينظر الى الاستكشاف كمملية غير فعالة، مع مدخل التنابع هناك العودة الى فترات استكشاف يتبعها الاستغلال،العملية الاستكشافية لديها الكثير من التجريب ويسبق عملية التكرار المتثاثل من الاستغلال. ان المدخل التنابعي هو ملائم اكثر لبيئة مستقرة وملائمة التي تتسم بفترات طويلة من الاستقرار قبل ان تضطر الى لى معالجة تغير كير، وفي المدخل المتزامن تعتبر التيار الرئيسي من الاستغلال والاستكشاف تيار الانشطة التي يعزز كل منها الاخر ، والتي يجب ان تحدث في الوقت نفسه، ويقوم هذا التعزيز على التعلم المتبادل بين الاثثين، وبالتالي المنظمة المتعلمة تنمو بسهولة اكثر ، والمدخل المتزامن يتلائم مع البيئات الديناميكية اكثر حسب الظروف المتغيرة باستمرار ، والمنظلات التي تثنافس في هذه الاسواق لم يكن لديهم الوقت للتحول من الاستكشاف الى الاستغلال لأن الفرصة المثاحة قصيرة جداً (Tushman \& O'Reilly, 2011: 14).

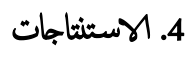
يوجد أهتام من الباحثين والكناب بشأن منافع تحقيق التوازن بين الاستغلال والاستكشاف ولكنها معقدة وصعبة التحقيق. 


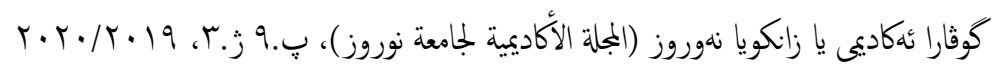

Organization and Strategy,Master thesis, Tilburg University,Holland.

3. Jansen, Justin, (2005), Ambidextrous Organizations, a multiplelevel study of absorptive capacity, exploratory and exploitative innovation and, performance, phd thesis, erasmus research institute of management (Erim), Erasmus University Rotterdam., Holland.

4. Yigit, Mert, (2013), Organizational Ambidexterity: Balancing Exploitation and Exploration in Organization, ,Master thesis, Entrepreneurship and Business Development, School of Management, Blekinge Institute of Technology, sweden.

5. Andriopoulos, Constantine., and Lewis, Marianne W., (2009). Exploitation-exporation tensions and organizational ambidexterity: Managing paradoxes of innovation. Organization Science, Vol. 20, No. 4.

6. Benner, M. J., Tushman, M. L. (2003), Exploitation, exploration, and process management: The productivity dilemma revisited, Academy of Management Review, Vol. 2, No. 28.

7. Bodwell, Wendy, (2011), A Theoretical model of organizational ambidexterity in hospitals, dissertation, school of education, in partial fulfillment of the requirements, for the degree of doctor of philosophy, Colorado State University, fort collins, Colorado.

8. Bot, S.D. (2012), Process Ambidexterity for Entrepreneurial Firms, journal of Technology Innovation Management Review

9. Bunge, M, (2004), How does it work? The search for explanatory mechanisms, Philosophy of the Social Sciences, Vol. 34, No. 2.

10. Datta, Avimanyu, (2011), Review and Extension on Ambidexterity: A Theoretical Model Integrating Networks and Absorptive Capacity, Journal of Management and Strategy, Vol. 2, No. 1.

11. Floyd, S., and Lane, P. (2000), Strategizing throughout the organization: Managing role conflict in strategic renewal. Academy of Management Review, Vol. 25.

12. Gibson, C.B., and Birkinshaw, J. (2004), The Antecedents, Consequences, and Mediating Role of Organizational Ambidexterity. Academy of Management, Journal, Vol. 47, No.2.

13. Gupta, A. K., Smith, K. G. and Shalley, C. E. (2006), The Interplay between Exploration and Exploitation, Academy of Management Journal, Vol. 49, No. 4.

14. He, Z.-L., and Wong, P.-K. (2004), Exploration vs. Exploitation: An Empirical Test of the Ambidexterity Hypothesis, Journal of Organization Science, Vol. 15, No. 4.

15. Holmqvist, M., (2004), Experiential learning processes of exploration and exploitation within and between organizations, An
ضرورة قيام المنظلات الحكومية في الأقليم بالاستغلال للموارد المتاحة و من ثخ الاستكشاف مستتقبلا.

لجوء المنظلات الى استخدام الفصل المهاني اي لايشترط قيام المنظمة بانشطة الاستغلال في جميع عملياتها، فقد يمكن ان تقوم بالاستغلال في الادارة الوسطى، و القيام بالاستكشاف في الادارة العليا .

ضرورة لجوء المنظلات او ادارات المنظلات الحكومية في الأقليم الى ايجاد توتزنات بين الاستكشاف و الاستغلال من خلال الاستخدام الامثل لجميع الموارد المالية و الموارد المعنوية الهخى .

استخدام الحوافز المشتركة وسياسات الموارد البشرية، مثل تعويض متغير يعتمد على أداء كل من أنشطة الاستغلال والاستكشاف .

ضرورة قيام المنظلت بالتكيف السرع مع المتغيرات البيئية المعقدة حاليا و كذلك محدودية الموارد. الاستعانة بصادر خارجية و التعاون مع القطاع الخاص.

عدم الاستغراق طويلا في حل التناقض لدى الحكومة و كفية اختيار احدها انما ايجاد طريقة لحل التناقض واستخدام الامثل او الاثنين معا.

استخدام الحلول الهيكلية التي من خلالها يمكن تنفيذ نشاطين في الوحدات التنظيمية و الدوائر الحكومية المختلفة.

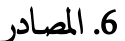

1.6 باللغة العربية

1. شرفاني، دلمان خوشفي رمضان، (2015)، تأثير البراعة التنظيمة في تحسين أبعاد جودة

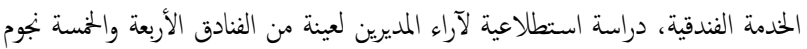

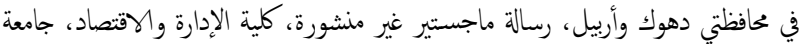

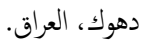

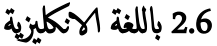

1. Bodwell, Wendy, (2011), A Theoretical model of organizational ambidexterity in hospitals, dissertation, school of education, in partial fulfillment of the requirements, for the degree of doctor of philosophy, Colorado State University, fort collins, Colorado

2. Bruijn, Edwin De (E.A.G), how to achieve Contextual Ambidexterity, (2011), Strategic Management, Department of 


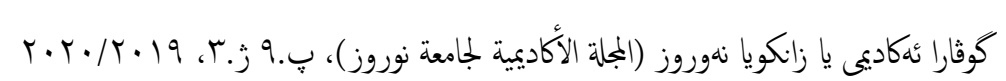

28. Smith, W.K. and Tushman, Michael L., (2005), Managing Strategic Contradictions: A Top Management Model for Managing Innovation Streams, Journal of Organization Science, Vol. 165.

29. Surendra, Bhusal, Dimitar, Korkov and Kaveh, Zadeh Sedigh, (2012), Ambidexterity and Success In the Swedish Construction Industry, Jönköping International Business School, Journal of Jönköping University, Sweden.

30. Szulanski, Martin \& Wiener, Martin, (2013), Challenges and Dilemmas in Open Innovation: Ambidexterity as Management Approach, University of Erlangen-Nuremberg, Chair of Information Systems III, Nuremberg, Germany, 11th International Conference on Wirtschaftsinformatik, 27th February - 01st March Leipzig, Germany. empirical study of product development, Journal of Organization science, Vol. 15.

16. Karrer, Daniel and Fleck, Denise Lima, (2013), Theoretical Explorations into Organizational Ambidexterity: Enabling the Construct's Exploitation in Practice, Enanpad, XXXVii Encontro do ANPAD, Rio do Joneiro/ RJ- 7 a 11 de setembro de Organization Science, Vol. 18, No. 4.

17. Lubatkin, M. H., Simsek, Z., Ling, Y., and Veiga, J. F. (2006), Ambidexterity and performance in small - to medium-sized firms: The pivotal role of TMT behavioral integration, Journal of Management, Vol. 32, No. 5.

18. Luscher L. and Lewis, M. (2008), Organizational change and managerial sensemaking: Working through paradox. Academy of Management Journal, Vol. 51.

19. O'Reilly C.A. and Tushman (2011), Organizational Ambidexterity in Action: How Managers Explore and Exploit, California Management Review, Vol. 53, No. 4.

20. O'Reilly, C. A., and Tushman, M. L. (2013c), Organizational ambidexterity: Past, present and future Academy of Management Perspectives (in press), Stanford Research Paper Series No. 2130.

21. Okhuisen, G.A. and K.M. Eisenhardt (2002), Integrating knowledge in groups: how formal, interventions enable flexibility, Journal of Organization Science, Vol. 13, No. 4.

22. O'Reilly, C. A. and Tushman, M. L. (2004), The Ambidextrous Organization, Harvard business review, Vol. 82, No. 4.

23. Puranam, Pranish, Harbir Singh, and Maurizio Zollo, (2006), Organizing for Innovation: Managing the CoordinationAutonomy Dilemma in Technology Acquisitions, Academy of Management Journal, Vol. 49.

24. Raisch, S., Birkinshaw, J. (2008), Organizational Ambidexterity: Antecendents, outcomes, and moderators. Journal of Management, Vol. 34, No. 3.

25. Sheremata, W. A. (2000), Centrifugal and Centripetal Forces in Radical New Product Development Under Time Pressure, Academy of Management Review, Vol. 25, No. 2, PP: 389-408

26. Simsek, Z. (2009), Organizational Ambidexterity: Towards a multilevel understanding. Journal of Management Studies, Vol. 46, No. 4.

27. Smith, W. K., and Lewis, M. W. (2011), Toward a Theory of Paradox: A Dynamic Equilibrium Model of Organizing, Academy of Management Review, Vol. 36, No. 2. 